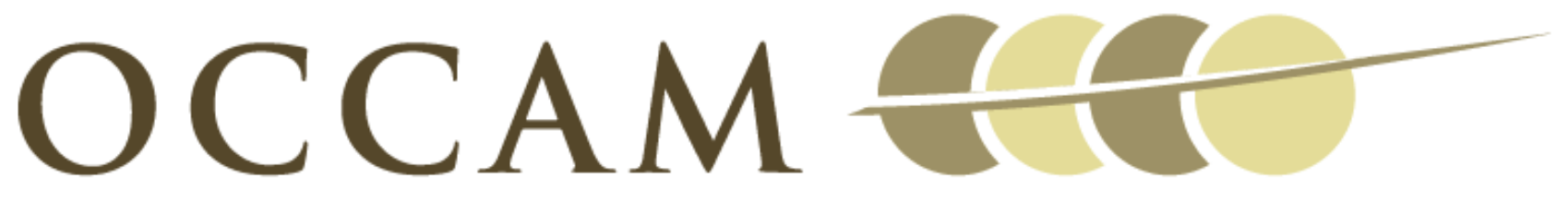

OXFORD CENTRE FOR COLLABORATIVE APPLIED MATHEMATICS

Report Number 10/23

Asymptotic analysis of a system of algebraic equations arising in dislocation theory

by

Cameron L. Hall, S. Jonathan Chapman and John R. Ockendon

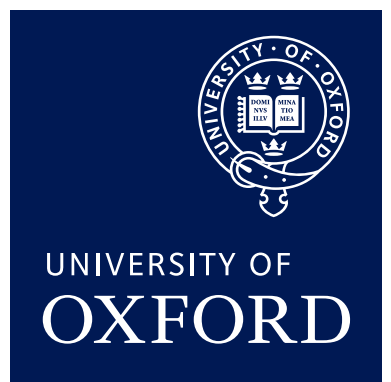

Oxford Centre for Collaborative Applied Mathematics Mathematical Institute 24 - 29 St Giles'

Oxford

OX1 3LB

England 



\title{
Asymptotic analysis of a system of algebraic equations arising in dislocation theory
}

\author{
Cameron L. Hall, S. Jonathan Chapman and John R. Ockendon
}

November 30, 2009

\begin{abstract}
The system of algebraic equations given by

$$
\begin{gathered}
\sum_{j=0, j \neq i}^{n} \frac{\operatorname{sgn}\left(x_{i}-x_{j}\right)}{\left|x_{i}-x_{j}\right|^{a}}=1, \quad i=1,2, \ldots, n, \\
x_{0}=0,
\end{gathered}
$$
\end{abstract}

appears in dislocation theory in models of dislocation pile-ups. Specifically, the case $a=1$ corresponds to the simple situation where $n$ dislocations are piled up against a locked dislocation, while the case $a=3$ corresponds to $n$ dislocation dipoles piled up against a locked dipole.

We present a general analysis of systems of this type for $a>0$ and $n$ large. In the asymptotic limit $n \rightarrow \infty$, it becomes possible to replace the system of discrete equations with a continuum equation for the particle density. For $0<a<2$, this takes the form of a singular integral equation, while for $a>2$ it is a first-order differential equation. The critical case $a=2$ requires special treatment but, up to corrections of logarithmic order, it also leads to a differential equation.

The continuum approximation is only valid for $i$ not too small nor too close to $n$. The boundary layers at either end of the pile-up are also analysed, which requires matching between discrete and continuum approximations to the main problem. 


\section{Introduction}

Dislocations are line defects in the crystal structure of a metal or similar crystalline material. They are of practical interest to material scientists and engineers because their motions and interactions are associated with important macroscopic phenomena such as plasticity, workhardening and crack propagation. When a dislocation source is located near an obstacle, pile-ups of dislocations can form [3]. A dislocation pile-up in equilibrium can be modelled by considering the balance between the forces of repulsion between dislocations and other externally-applied forces. Although models of this form have a long history in dislocation mechanics (see, for example, the discussion in Hirth and Lothe [3]), it is only recently that the asymptotic analysis of a pile-up of a large number of dislocations has been considered. Most notably, Voskoboinikov and coworkers $[4,5,6]$ have used asymptotic techniques to analyse pile-ups against locked dislocations and against bimetallic interfaces.

Similar to these pile-ups of dislocation monopoles, it is possible to observe 'ladders' of dislocation dipoles in some materials [1]. A pile-up of a large number of dislocation dipoles against a locked dipole leads to a system of equations that is very similar to the system of equations for a pile-up of dislocation monopoles, the only difference being that the repulsive force between dipoles is inversely proportional to the cube of distance while the repulsive force between monopoles is inversely proportional to distance. The asymptotic analysis of a pile-up of dipoles is, however, qualitatively very different from the analysis of a pile-up of monopoles. Monopole pile-ups are dominated by non-local effects, leading to a singular integral equation for the monopole density. In contrast, dipole pile-ups are dominated by short-range repulsion, leading to a differential equation for the dipole density.

In this paper, we consider a pile-up of $n$ particles where the force of repulsion between them is inversely proportional to the $a$-th power of distance. From this analysis, we can construct a mathematical bridge between the asymptotic behaviour of a pile-up of dislocation monopoles and a pile-up of dislocation dipoles. 


\section{Problem statement}

We consider the following system of $n$ algebraic equations for unknowns $x_{i}$ :

$$
\sum_{j=0, j \neq i}^{n} \frac{\operatorname{sgn}\left(x_{i}-x_{j}\right)}{\left|x_{i}-x_{j}\right|^{a}}=1, \quad i=1,2, \ldots, n,
$$

subject to the condition

$$
x_{0}=0 \text {. }
$$

The solutions $\left\{x_{i}\right\}$ to this system can be thought of as representing the locations of $n$ mutually-repelling particles held in equilibrium by a constant external force with the particle at $x_{0}=0$ being fixed. We are interested in the asymptotic behaviour of the solution to (1) in the limit as $n \rightarrow \infty$. In particular, it is of interest to determine the locations of the particles nearest $x_{0}=0$ and the location of the final particle, $x_{n}$.

For large $n$, we expect that it will be appropriate to consider an averaged particle density rather than trying to find the location of each individual particle. However, the total space occupied by the particles will increase as $n$ increases and we need to take this into account before we can define a density function. Suppose there exists $q(a)$ such that $x_{n} \sim n^{q}$ as $n \rightarrow \infty$. Thus, we define a rescaled spatial variable, $\xi$, so that $x=\xi n^{q}$ and we write $x_{i}=\xi_{i} n^{q}$. All definitions of particle density will be given in terms of $\xi$, but we still need to determine the dependence of $q$ on $a$. We also define $\xi^{*}$ so that

$$
\xi^{*}=\lim _{n \rightarrow \infty} \xi_{n}=\lim _{n \rightarrow \infty} \frac{x_{n}}{n^{q}}
$$

Using the rescaled spatial variable, the system in (1) takes the form

$$
\begin{gathered}
n^{-a q} \sum_{j=0, j \neq i}^{n} \frac{\operatorname{sgn}\left(\xi_{i}-\xi_{j}\right)}{\left|\xi_{i}-\xi_{j}\right|^{a}}=1, \quad i=1,2, \ldots, n, \\
\xi_{0}=0 .
\end{gathered}
$$

We now proceed on the assumption that the gaps between neighbouring particles do not 
vary rapidly with $i$, except possibly near the ends of the pile-up. Hence, it is appropriate to propose a smooth particle density function to represent the distribution of the particles. We assume that this density function, $\rho(\xi ; n)$, will satisfy the following conditions. Firstly, $\rho(\xi ; n)$ will be defined for all $\xi>0$ with $\rho(\xi ; n)>0$ for $0<\xi<\xi_{n}$ and $\rho(\xi ; n)=0$ for $\xi \geq \xi_{n}$. As we will consider Taylor series representations of $\rho(\xi ; n)$ on $\left(0, \xi_{n}\right)$, we require $\rho$ to be analytic on this domain and as smoothly varying as possible; specifically, we exclude oscillations or other rapid changes in $\rho$ or its derivatives. Most importantly, we require that

$$
\int_{\xi_{i}}^{\xi_{i+1}} \rho(\xi ; n) d \xi=\frac{1}{n}, \quad i=0,1, \ldots, n-1
$$

For any given value of $n$, there will be a family of functions $\rho(\xi ; n)$ that satisfy the conditions stated above. However, as long as all the conditions are satisfied, it will be possible to recover the solutions of $(3)$ from $\rho(\xi ; n)$ by using $(4)$. Moreover, we expect that the particle density function will converge to some well-defined limit as $n \rightarrow \infty$; this will be the leading-order asymptotic density, $\rho_{0}(\xi)$. From equations (2) and (4), we note that

$$
\int_{0}^{\xi^{*}} \rho_{0}(\xi) d \xi=1
$$

and that

$$
\rho_{0}\left(\xi^{*}\right)=0
$$

Unless necessary for clarity, we will omit the dependence on $n$ and write $\rho(\xi ; n)$ as $\rho(\xi)$. From (4), it follows that

$$
\int_{\xi_{i}}^{\xi_{i+k}} \rho(\xi) d \xi=\frac{k}{n}
$$

Proposing a Taylor series for $\rho(\xi)$ around $\xi_{i}$ and expressing $\xi_{i+k}$ as a series in reciprocal powers of $n$, this can be used to obtain the following formula:

$$
\xi_{i+k}-\xi_{i}=\frac{k}{\rho\left(\xi_{i}\right)} n^{-1}-\frac{k^{2} \rho^{\prime}\left(\xi_{i}\right)}{2 \rho^{3}\left(\xi_{i}\right)} n^{-2}+\mathcal{O}\left(\frac{k^{3}}{n^{3}}\right)
$$


Similarly, we find that

$$
\xi_{i}-\xi_{i-k}=\frac{k}{\rho\left(\xi_{i}\right)} n^{-1}+\frac{k^{2} \rho^{\prime}\left(\xi_{i}\right)}{2 \rho^{3}\left(\xi_{i}\right)} n^{-2}+\mathcal{O}\left(\frac{k^{3}}{n^{3}}\right)
$$

These can be combined to yield the important result that

$$
\frac{1}{\left(\xi_{i}-\xi_{i-k}\right)^{a}}-\frac{1}{\left(\xi_{i+k}-\xi_{i}\right)^{a}}=-\frac{a \rho^{\prime}\left(\xi_{i}\right) \rho^{a-2}\left(\xi_{i}\right)}{k^{a-1}} n^{a-1}+\mathcal{O}\left(\frac{n^{a-2}}{k^{a-2}}\right) .
$$

We will make extensive use of this result in the following sections.

\section{Leading-order asymptotic analysis}

\subsection{Case 1: $0<a<2$}

In the case where $0<a<2$, we can rearrange (3a) to recover, at leading order, an integral equation. To see this, we first split the sum into two parts:

$$
n^{-a q} \sum_{j=0}^{i-1} \frac{1}{\left(\xi_{i}-\xi_{j}\right)^{a}}-n^{-a q} \sum_{j=i+1}^{n} \frac{1}{\left(\xi_{j}-\xi_{i}\right)^{a}}=1
$$

Applying the Euler-Maclaurin summation formula [2], this yields

$$
\begin{aligned}
n^{1-a q}\left(\int_{0}^{\xi_{i-1}}\right. & \left.\frac{\rho\left(\xi^{\prime}\right)}{\left(\xi_{i}-\xi^{\prime}\right)^{a}} d \xi^{\prime}-\int_{\xi_{i+1}}^{\xi_{n}} \frac{\rho\left(\xi^{\prime}\right)}{\left(\xi^{\prime}-\xi_{i}\right)^{a}} d \xi^{\prime}\right) \\
& +n^{-a q}\left(\frac{1}{2 \xi_{i}^{a}}+\frac{1}{2\left(\xi_{i}-\xi_{i-1}\right)^{a}}-\frac{1}{2\left(\xi_{i+1}-\xi_{i}\right)^{a}}-\frac{1}{2\left(\xi_{n}-\xi_{i}\right)^{a}}+\ldots\right) \sim 1 .
\end{aligned}
$$

Using (6), it follows that

$$
n^{-a q}\left(\frac{1}{2\left(\xi_{i}-\xi_{i-1}\right)^{a}}-\frac{1}{2\left(\xi_{i+1}-\xi_{i}\right)^{a}}\right) \sim-a \frac{\rho^{\prime}\left(\xi_{i}\right)}{2 \rho^{2-a}\left(\xi_{i}\right)} n^{a-a q-1}+\mathcal{O}\left(n^{a-a q-2}\right) .
$$


Assuming $\left(\xi_{i}\right)^{-a}$ and $\left(\xi_{n}-\xi_{i}\right)^{-a}$ to be order-one terms, we thus find that

$$
n^{1-a q}\left(\int_{0}^{\xi_{i-1}} \frac{\rho\left(\xi^{\prime}\right)}{\left(\xi_{i}-\xi^{\prime}\right)^{a}} d \xi^{\prime}-\int_{\xi_{i+1}}^{\xi_{n}} \frac{\rho\left(\xi^{\prime}\right)}{\left(\xi^{\prime}-\xi_{i}\right)^{a}} d \xi^{\prime}\right)+\mathcal{O}\left(n^{r}\right)=1
$$

where $r=\max [a-1-a q,-a q]$. Note that $\left(\xi_{i}\right)^{-a}$ and $\left(\xi_{n}-\xi_{i}\right)^{-a}$ will not be order one if $i$ is close to zero or close to $n$, leading us to expect boundary layers at both ends of our domain.

When $0<a<2$, we find that it is only by taking $q=\frac{1}{a}$ that we achieve a leading-order balance in (7) between the integral terms on the left-hand side and the constant term on the right-hand side. As $a-1-a q=a-2$ is negative in this case, the $\mathcal{O}\left(n^{r}\right)$ terms in (8) can always be treated as a small correction. Moreover, we find the boundary layers occur when $\xi \sim n^{-\frac{1}{a}}$ and when $\left(\xi^{*}-\xi\right) \sim n^{-\frac{1}{a}}$.

As $n \rightarrow \infty$, the leading-order equation for the particle density is given by

$$
\lim _{\epsilon \rightarrow 0}\left[\int_{0}^{\xi-\epsilon} \frac{\rho_{0}\left(\xi^{\prime}\right)}{\left(\xi-\xi^{\prime}\right)^{a}} d \xi^{\prime}-\int_{\xi+\epsilon}^{\xi^{*}} \frac{\rho_{0}\left(\xi^{\prime}\right)}{\left(\xi^{\prime}-\xi\right)^{a}} d \xi^{\prime}\right]=1, \quad 0<\xi<\xi^{*}
$$

or equivalently,

$$
f_{0}^{\xi^{*}} \frac{\operatorname{sgn}\left(\xi-\xi^{\prime}\right)}{\left|\xi-\xi^{\prime}\right|^{a}} \rho_{0}\left(\xi^{\prime}\right) d \xi^{\prime}=1, \quad 0<\xi<\xi^{*}
$$

where the integral is interpreted in the normal sense for $0<a<1$, as a Cauchy principal value integral for $a=1$ and as a Hadamard finite part integral for $1<a<2$.

When $a=1$, equation (9) is the Cauchy integral equation discussed by Voskoboinikov et al. [6] for a pile-up of dislocation monopoles. This integral equation has a closed-form solution, but we have not been able to find closed-form solutions for the case of general $a \in(0,2)$.

If $a \geq 2$, however, the terms of relative order $n^{-(2-a)}$ in (7) are no longer negligible. Importantly, it can be shown that all the non-integral terms in the Euler-Maclaurin series are of this size, with the result that we cannot terminate the series at any point to obtain the leading-order equation. 


\subsection{Case 2: $a>2$}

When $a>2$, we find that the dominant contribution to the force on a particle comes from its nearest neighbours. However, the left-hand neighbour and right-hand neighbour push in opposite directions with almost equal force, making it useful to pair them instead of treating them separately. In order to obtain the equation for $a>2$, we therefore rearrange the sum in (3a) by taking the particles around $\xi_{i}$ in pairs until a boundary is reached. Without loss of generality, let $i \leq \frac{n}{2}$; then, (3a) becomes

$$
n^{-a q} \sum_{k=1}^{i}\left(\frac{1}{\left(\xi_{i}-\xi_{i-k}\right)^{a}}-\frac{1}{\left(\xi_{i+k}-\xi_{i}\right)^{a}}\right)-n^{-a q} \sum_{j=2 i+1}^{n} \frac{1}{\left(\xi_{j}-\xi_{i}\right)^{a}}=1 .
$$

From (6), we find that

$$
n^{-a q} \sum_{k=1}^{i}\left(\frac{1}{\left(\xi_{i}-\xi_{i-k}\right)^{a}}-\frac{1}{\left(\xi_{i+k}-\xi_{i}\right)^{a}}\right)=\sum_{k=1}^{i} \frac{-a \rho^{\prime}\left(\xi_{i}\right) \rho^{a-2}\left(\xi_{i}\right)}{k^{a-1}} n^{a-1-a q}+\mathcal{O}\left(n^{a-2-a q} i^{s}\right)
$$

where $s=\max [0,3-a]$. This indicates that the appropriate balance will only be achieved when $q=\frac{a-1}{a}$. Moreover, we note that the finite sum in (11) can be approximated with an infinite one, yielding

$$
\sum_{k=1}^{i} \frac{-a \rho^{\prime}\left(\xi_{i}\right) \rho^{a-2}\left(\xi_{i}\right)}{k^{a-1}}=-a \zeta(a-1) \rho_{0}^{\prime}(\xi) \rho_{0}{ }^{a-2}(\xi)+\mathcal{O}\left(i^{-(a-2)}\right)
$$

The other sum in (10) can be approximated using Euler-Maclaurin series. Thus,

$$
n^{-(a-1)} \sum_{j=2 i+1}^{n} \frac{1}{\left(\xi_{j}-\xi_{i}\right)^{a}}=n^{-(a-2)} \int_{\xi_{2 i+1}}^{\xi_{n}} \frac{\rho\left(\xi^{\prime}\right)}{\left(\xi^{\prime}-\xi_{i}\right)^{a}} d \xi^{\prime}+\mathcal{O}\left(n i^{-a}\right) .
$$

These terms will be negligible when $i$ is large, but they cannot be ignored for particles near the boundary. Thus, (13) indicates that we expect boundary layer behaviour when $i \sim n^{\frac{1}{a}}$ (that is, when $\xi \sim n^{-\frac{a-1}{a}}$ ). 
For $\xi_{i}$ away from the boundary layer, we ultimately find that (11), (12) and (13) combine to give

$$
-a \zeta(a-1) \rho_{0}^{\prime}\left(\xi_{i}\right) \rho_{0}{ }^{a-2}\left(\xi_{i}\right)+\mathcal{O}\left(n^{u}\right)=1
$$

where $u=\max [-1,-(a-2)]$.

In the limit $n \rightarrow \infty$, we then recover the leading-order density equation:

$$
-a \zeta(a-1) \rho_{0}^{\prime}(\xi) \rho_{0}^{a-2}(\xi)=1
$$

Using (5) to supply the needed boundary condition, (15) yields

$$
\xi^{*}=\frac{a \zeta(a-1)^{\frac{1}{a}}}{a-1}
$$

and thus,

$$
\rho_{0}(\xi)=\left(\frac{1}{\zeta(a-1)^{\frac{a-1}{a}}}-\frac{a-1}{a \zeta(a-1)} \xi\right)^{\frac{1}{a-1}}
$$

Note that (15) will not be valid in the boundary layers where $i \sim n^{\frac{1}{a}}$ or $(n-i) \sim n^{\frac{1}{a}}$. These will be considered in Section 5 .

\subsection{Case 3: $a=2$}

By combining the $0<a<2$ and $a>2$ approaches, it is possible to obtain an equation for the leading-order particle density when $a=2$. Firstly, we note that the scaling $x=\xi n^{\frac{1}{a}}$ was appropriate when $0<a<2$, while the scaling $x=\xi n^{\frac{a-1}{a}}$ was appropriate when $a>2$. This suggests that the correct scaling for $a=2$ will be $x=\xi n^{\frac{1}{2}}$. Although we will find that this is not entirely correct, $x=\xi n^{\frac{1}{2}}$ can be used as a starting point in our attempt to find the leading-order density equation.

Taking $a=2$ and $q=\frac{1}{2}$, we split the sum in (3a) into a term arising from particles in the 
neighbourhood of $\xi_{i}$ and other terms representing more distant particles. Thus,

$$
n^{-1} \sum_{k=1}^{M-1}\left(\frac{1}{\left(\xi_{i}-\xi_{i-k}\right)^{2}}-\frac{1}{\left(\xi_{i+k}-\xi_{i}\right)^{2}}\right)+n^{-1} \sum_{j=0}^{i-M} \frac{1}{\left(\xi_{i}-\xi_{j}\right)^{2}}-n^{-1} \sum_{j=i+M}^{n} \frac{1}{\left(\xi_{j}-\xi_{i}\right)^{2}}=1
$$

where $M(i ; n)$ represents the size of the neighbourhood being treated as the local contribution to the force balance and is yet to be specified. We note, however, that $M \leq \min [i, n-i]$.

Using (6) to simplify the first term and using Euler-Maclaurin series to simplify the other sums, we find that

$$
\begin{aligned}
& -2 \rho^{\prime}\left(\xi_{i}\right) \sum_{k=1}^{M-1} \frac{1}{k}+\int_{0}^{\xi_{i-M}} \frac{\rho\left(\xi^{\prime}\right)}{\left(\xi_{i}-\xi^{\prime}\right)^{2}} d \xi^{\prime}-\int_{\xi_{i+M}}^{\xi_{n}} \frac{\rho\left(\xi^{\prime}\right)}{\left(\xi^{\prime}-\xi_{i}\right)^{2}} d \xi^{\prime} \\
& +n^{-1}\left(\frac{1}{2 \xi_{i}^{2}}+\frac{1}{2\left(\xi_{i}-\xi_{i-M}\right)^{2}}-\frac{1}{2\left(\xi_{i+M}-\xi_{i}\right)^{2}}-\frac{1}{2\left(\xi_{n}-\xi_{i}\right)^{2}}+\ldots\right)+\mathcal{O}\left(M n^{-1}\right)=1 .
\end{aligned}
$$

We now combine the integrals in (18) by defining the singular integral

$$
\begin{aligned}
& \int_{0}^{\xi^{*}} \frac{\operatorname{sgn}\left(x_{0}-x\right)}{\left(x_{0}-x\right)^{2}} \phi(x) d x \\
& \quad=\lim _{\epsilon \rightarrow 0}\left[\int_{0}^{x_{0}-\epsilon} \frac{\phi(x)}{\left(x_{0}-x\right)^{2}} d x-\int_{x_{0}+\epsilon}^{\xi^{*}} \frac{\phi(x)}{\left(x_{0}-x\right)^{2}} d x-2 \phi^{\prime}\left(x_{0}\right) \log \epsilon+2 \phi^{\prime}\left(x_{0}\right)\right] .
\end{aligned}
$$

Hence,

$$
\begin{aligned}
\int_{0}^{\xi_{i-M}} & \frac{\rho\left(\xi^{\prime}\right)}{\left(\xi_{i}-\xi^{\prime}\right)^{2}} d \xi^{\prime}-\int_{\xi_{i+M}}^{\xi_{n}} \frac{\rho\left(\xi^{\prime}\right)}{\left(\xi^{\prime}-\xi_{i}\right)^{2}} d \xi^{\prime} \\
& =f_{0}^{\xi_{n}} \frac{\operatorname{sgn}\left(\xi_{i}-\xi^{\prime}\right)}{\left(\xi_{i}-\xi^{\prime}\right)^{2}} \rho\left(\xi^{\prime}\right) d \xi^{\prime}+2 \rho^{\prime}\left(\xi_{i}\right) \log \left(\frac{M}{n \rho\left(\xi_{i}\right)}\right)-2 \rho^{\prime}\left(\xi_{i}\right)+\mathcal{O}\left(M n^{-1}\right)
\end{aligned}
$$

Also, we can use (6) to show that

$$
n^{-1}\left(\frac{1}{2\left(\xi_{i}-\xi_{i-M}\right)^{2}}-\frac{1}{2\left(\xi_{i+M}-\xi_{i}\right)^{2}}\right)=\mathcal{O}\left(M^{-1}\right) .
$$


Lastly, we note that

$$
n^{-1} \frac{1}{2 \xi_{i}^{2}}=\mathcal{O}\left(n i^{-2}\right)
$$

with a similar result holding for $\xi_{n}-\xi_{i}$. In the outer region where $i \sim n$, these terms will both be $\mathcal{O}\left(n^{-1}\right)$. However, they indicate that we expect to see boundary layers when $i \sim n^{\frac{1}{a}}$ or $(n-i) \sim n^{\frac{1}{a}}$.

Substituting (19) to (21) into (18) and using the fact that

$$
\sum_{k=1}^{M-1} \frac{1}{k}=\log (M)+\gamma+\mathcal{O}\left(M^{-1}\right)
$$

we find that the minimum error is obtained when $M \propto n^{\frac{1}{2}}$ and that

$$
\begin{aligned}
-2 \gamma \rho^{\prime}\left(\xi_{i}\right)+f_{0}^{\xi_{n}} \frac{\operatorname{sgn}\left(\xi_{i}-\xi^{\prime}\right)}{\left(\xi_{i}-\xi^{\prime}\right)^{2}} \rho\left(\xi^{\prime}\right) d \xi^{\prime}-2 \rho^{\prime}\left(\xi_{i}\right) \log n-2 \rho^{\prime}\left(\xi_{i}\right) \log \left(\rho\left(\xi_{i}\right)\right) & \\
& -2 \rho^{\prime}\left(\xi_{i}\right)+\mathcal{O}\left(n^{-\frac{1}{2}}\right)=1
\end{aligned}
$$

Unfortunately, the $\log n$ term always dominates the left-hand side of (22), which means that we have not used the correct scaling for $\xi$. However, if we were to take $-2 \rho^{\prime}(\xi) \log n=1$ to be the leading-order equation arising from (22), we could easily obtain a solution subject to the boundary conditions from (5). This indicates that $\xi_{n} \sim(\log n)^{\frac{1}{2}}$ with the current scaling. Hence, in order for $\xi_{n}$ to tend to a finite limit as $n \rightarrow \infty$, the correct rescaling is $\xi=\tilde{\xi}(\log n)^{\frac{1}{2}}$, or equivalently, $x=\tilde{\xi}(n \log n)^{\frac{1}{2}}$.

Applying this rescaling to the original problem and writing $\tilde{\xi}$ as $\xi$, we find that

$$
\begin{array}{r}
-2 \rho^{\prime}\left(\xi_{i}\right)+\frac{1}{\log n}\left[-2 \gamma \rho^{\prime}\left(\xi_{i}\right)+f_{0}^{\xi_{n}} \frac{\operatorname{sgn}\left(\xi_{i}-\xi^{\prime}\right)}{\left(\xi_{i}-\xi^{\prime}\right)^{2}} \rho\left(\xi^{\prime}\right) d \xi^{\prime}-2 \rho^{\prime}\left(\xi_{i}\right) \log \left(\rho\left(\xi_{i}\right)\right)-2 \rho^{\prime}\left(\xi_{i}\right)\right] \\
+\mathcal{O}\left(n^{-\frac{1}{2}}\right)=1 .
\end{array}
$$

In the limit $n \rightarrow \infty$, this gives

$$
-2 \rho_{0}^{\prime}(\xi)=1
$$


and, using (5),

$$
\rho_{0}(\xi)=1-\frac{1}{2} \xi
$$

From this, we find that $\xi^{*}=2$ for $a=2$. In Section 4 , these results will be compared with numerical solutions for the full problem.

\section{Numerical solution to the original system}

The original system in (1) can be solved numerically using Newton's method. For $a \geq 2$, it is possible to integrate (17) or (25) to obtain asymptotic expressions for $x_{i}$ that would work well as starting points for this method. However, we wish to develop a numerical scheme that is valid for all $a$.

Hence, to obtain an initial guess for $x_{i}$ we consider the simplified problem given by

$$
\begin{gathered}
\frac{1}{\left(x_{i}-x_{i-1}\right)^{a}}-\frac{1}{\left(x_{i+1}-x_{i}\right)^{a}}=1, \quad i=1,2, \ldots, n-1, \\
\frac{1}{\left(x_{i}-x_{i-1}\right)^{a}}=1, \quad i=n .
\end{gathered}
$$

This corresponds to the case where each particle is only affected by its nearest neighbours. The system in (26) can be solved explicitly to give

$$
x_{i}-x_{i-1}=(n+1-i)^{-\frac{1}{a}}
$$

which we use as our starting point all values of $a$. The solver is constructed so that the unknown variables are $\Delta x_{i}=x_{i}-x_{i-1}$ rather than $x_{i}$; this leads to a better-conditioned system.

The numerical solutions obtained for $x_{i}$ can be rescaled to give $\xi_{i}$. Given these solutions, we seek particle density functions, $\rho(\xi ; n)$, that are comparable with the leading-order asymptotic solution. Taking $\rho(\xi ; n)$ to be a piecewise constant function in $(4)$, we note that

$$
\rho(\xi ; n) \approx\left(\xi_{i}-\xi_{i-1}\right)^{-1} n^{-1}
$$


where $\xi \in\left(\xi_{i-1}, \xi_{i}\right)$. Hence, we can approximate $\rho(\xi ; n)$ by plotting $\left(\xi_{i}-\xi_{i-1}\right)^{-1} n^{-1}$ against $\frac{1}{2}\left(\xi_{i}+\xi_{i-1}\right)$. This is illustrated in Figures 1 and 2 for $a=3$ and $a=2$ respectively.

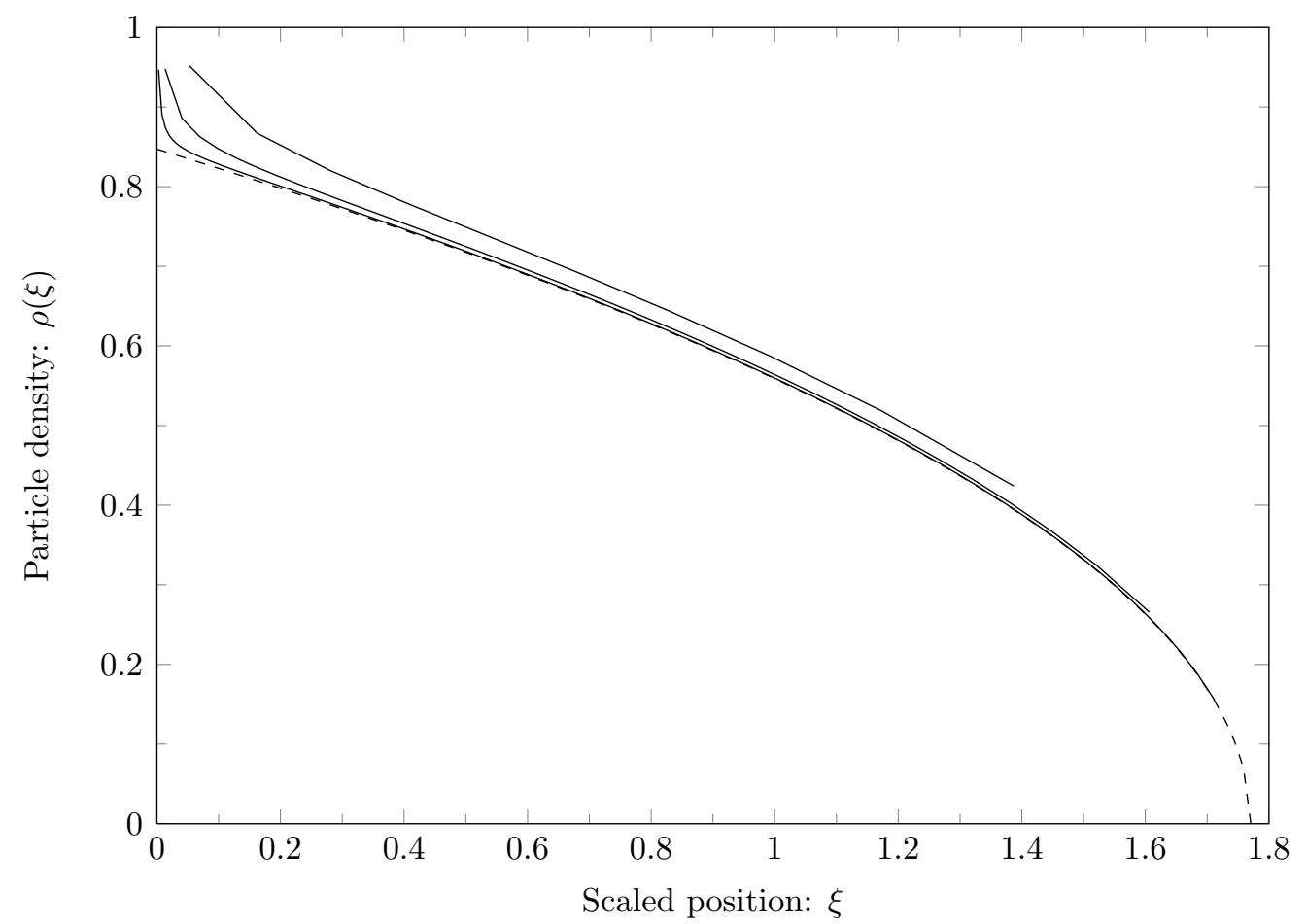

Figure 1: Numerically calculated particle densities for $a=3$ compared with the leading-order asymptotic density (dashed). From top to bottom, the continuous curves represent $n=10, n=$ 40 and $n=200$. In each case, the numerical density is given by $\left(\xi_{i}-\xi_{i-1}\right)^{-1} n^{-1}$, while the corresponding $\xi$ coordinate is given by $\left(\xi_{i}+\xi_{i-1}\right) / 2$.

Figures 1 and 2 clearly show that the numerical solution from the particle density deviates from the asymptotic prediction when $\xi$ is close to zero. This is the boundary layer that we expect since (15) and (23) are invalid for $i \sim n^{\frac{1}{a}}$. Although this boundary layer is more dramatic for $a=2$ than it is for $a=3$, we will see in Section 5 that the left-hand boundary layer has the same structure for all $a \geq 2$.

It is interesting to note that we also expect to see a boundary layer near $\xi^{*}$, but that it is not immediately apparent from Figures 1 and 2. One visible effect of the right-hand boundary layer is that $\xi_{n}$ is always considerably less than the theoretical $\xi^{*}$ obtained from equation (16). This is illustrated in Figure 3 and we will analyse this boundary layer further in Section 5.3 . 


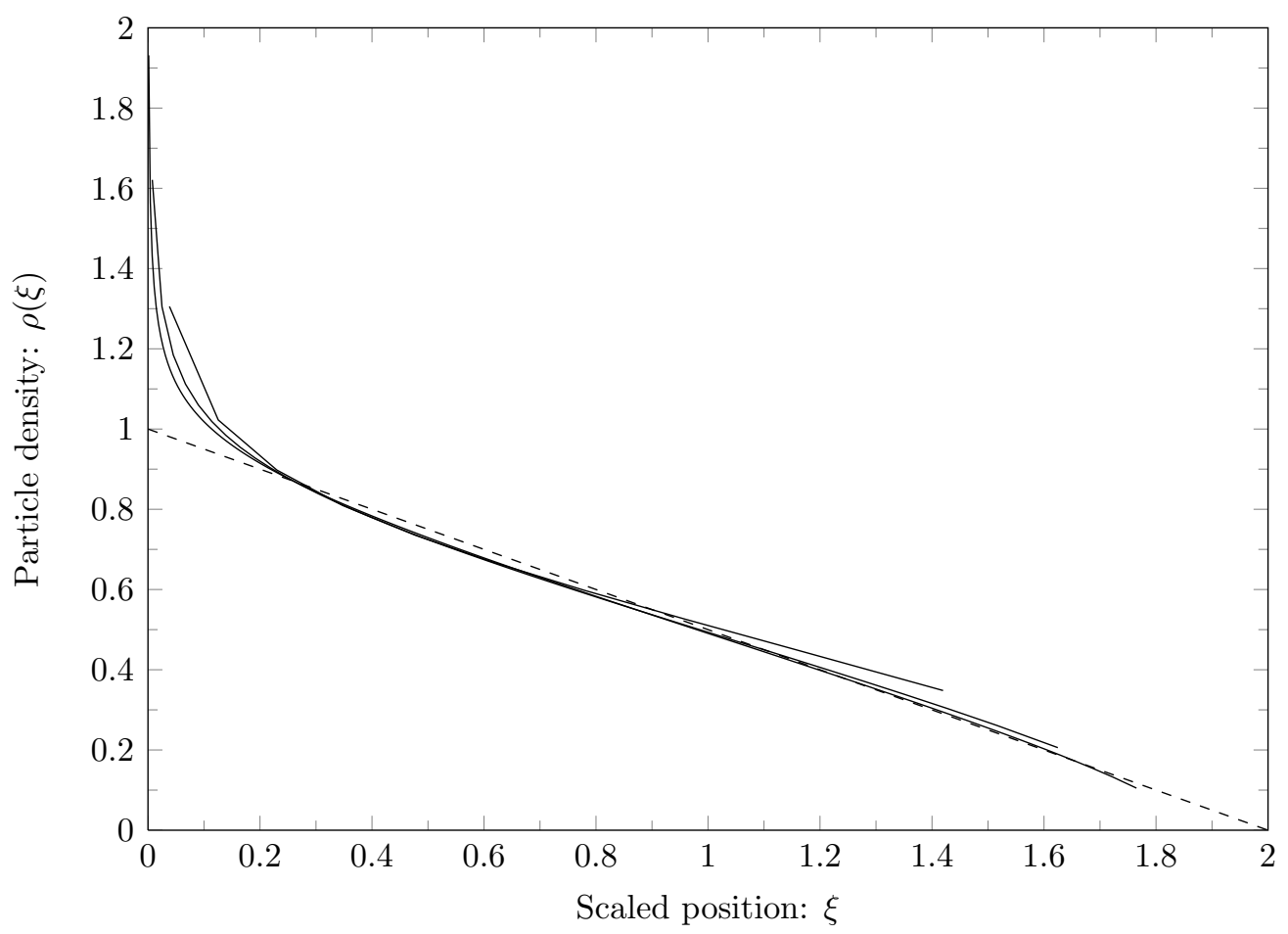

Figure 2: Numerically calculated particle densities for $a=2$ compared with the leading-order asymptotic density (dashed). From top to bottom, the continuous curves represent $n=10, n=$ 40 and $n=200$. In each case, the numerical density is given by $\left(\xi_{i}-\xi_{i-1}\right)^{-1} n^{-1}$, while the corresponding $\xi$ coordinate is given by $\left(\xi_{i}+\xi_{i-1}\right) / 2$. 


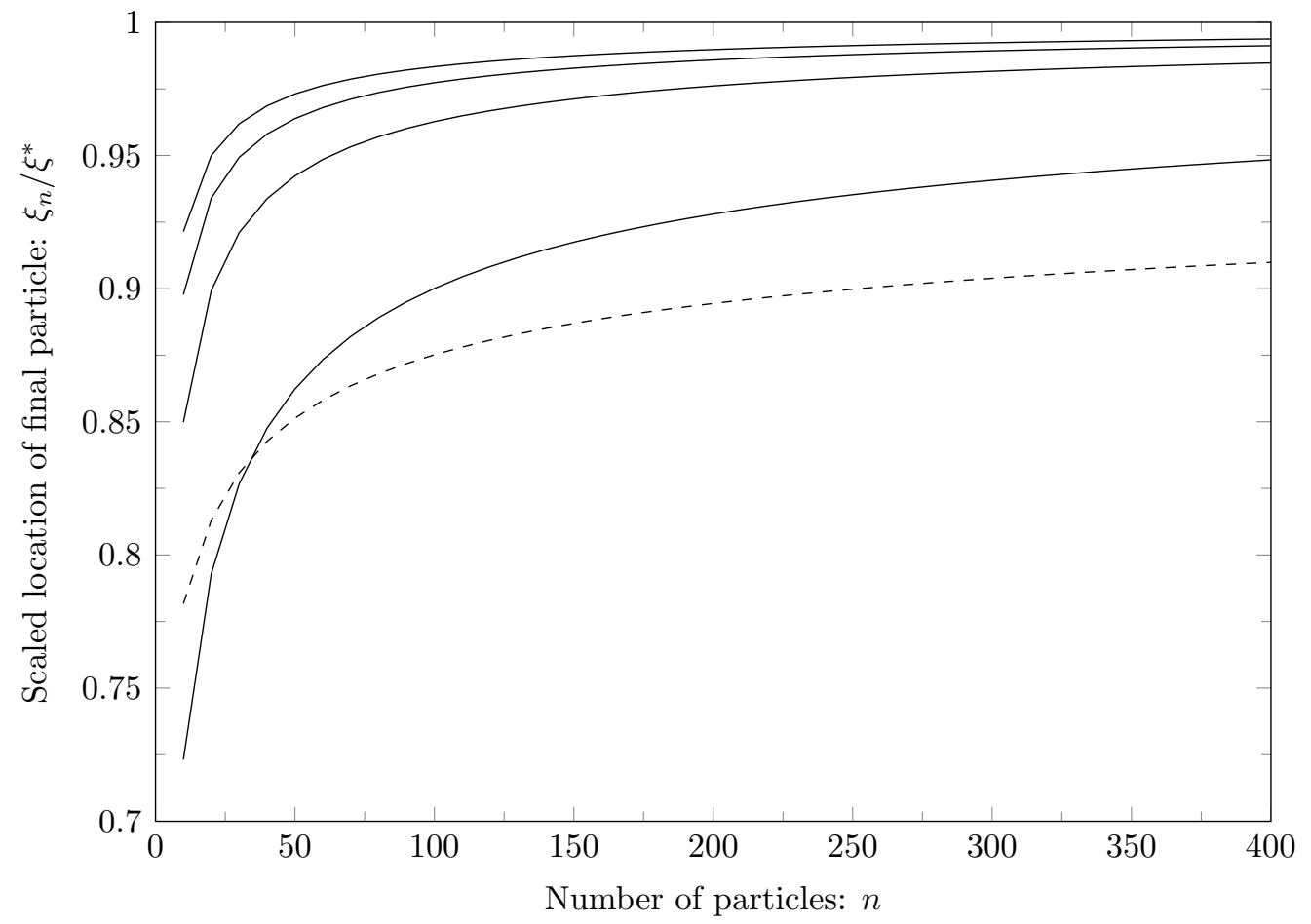

Figure 3: Ratio of numerically obtained final particle location, $\xi_{n}$, to predicted final particle location, $\xi^{*}$ for varying values of $n$. The results for $a=2$ are shown as a dashed line, while the solid lines (from bottom to top) represent $a=2.5, a=3, a=3.5$ and $a=4$. In each case, $\xi_{n} / \xi^{*}$ should tend to 1 as $n$ tends to infinity, but this happens quite slowly. Improved results can be obtained by considering the boundary layer near $\xi^{*}$. 
Lastly, we note that the numerical densities obtained when $a=2$ show more marked differences from the leading-order asymptotic density than those for $a=3$. This is to be expected, as the corrections to (24) are only logarithmically small, while those to (15) are algebraically small. Indeed, it can also be seen that the accuracy of (15) gets worse as $a \searrow 2$.

\section{$5 \quad$ Boundary layers}

Regardless of the value of $a$, the solution to (3) exhibits boundary layer behaviour at both ends of the domain of solution. However, the boundary layer near $\xi=0$ is very different from the boundary layer near $\xi=\xi^{*}$ and the boundary layers change in character as $a$ increases through two. Here, we present a detailed analysis of three of these different cases: the boundary layer near $\xi=0$ for $0<a<2$, the boundary layer near $\xi=0$ for $a>2$ and the boundary layer near $\xi=\xi^{*}$ for $a>2$.

We leave the problem of boundary layer analysis for $a=2$ as an open problem. In this case, (23) suggests that an expansion for $\rho(\xi)$ in powers of $\frac{1}{\log n}$ is appropriate. When using Van Dyke's matching rule, these are all taken to be order-one terms and it would be necessary to find them before attempting to match between the outer problem and the inner problem.

Interestingly, we can use (23) to obtain the first correction to (25). This indicates that $\rho(\xi)$ has a logarithmic singularity at $\xi=0$ and that $\rho^{\prime}(\xi)$ has a logarithmic singularity at $\xi=\xi^{*}$. As a result of these, it is not even straightforward to determine the appropriate rescaling in these boundary layers.

\subsection{Boundary layer near $\xi=0$ for $0<a<2$}

The fact that we do not have a general closed-form solution to the integral equation (9) also makes it difficult to analyse the boundary layers when $0<a<2$. However, useful results can still be obtained by making appropriate assumptions about the form of the outer solution and exploiting certain features of (1). 
When $a=1$, Voskoboinikov et al. [6] found that the solution of (9) gives $\rho(\xi) \sim \xi^{-\frac{1}{2}}$ as $\xi \rightarrow 0$. Similarly, it is reasonable to expect that the density will become singular at $\xi=0$ when $0<a<2$. Thus, we propose

$$
\rho=A \xi^{-\kappa}+o\left(\xi^{-\kappa}\right)
$$

for some $\kappa$ and $A$ to be determined with $0<\kappa<1$. Integrating (27) and using (4), we find that

$$
A \int_{0}^{\xi_{i}} \xi^{-\kappa} d \xi=i n^{-1}+o\left(\xi^{1-\kappa}\right)
$$

and hence

$$
\xi_{i}=B i^{\frac{1}{1-\kappa}} n^{-\frac{1}{1-\kappa}}+o\left(n^{-\frac{1}{1-\kappa}}\right)
$$

where $B=\left(\frac{1-\kappa}{A}\right)^{\frac{1}{1-\kappa}}$. Thus, it is appropriate to rescale our problem in the inner region by introducing a new spatial variable, $\eta$, such that $\xi=\eta n^{-\frac{1}{1-\kappa}}$ and hence $x=\eta n^{\frac{1}{a}-\frac{1}{1-\kappa}}$.

Now, it is useful to determine $\kappa$ before we consider the leading-order inner problem in greater depth. To find $\kappa$, we first add all $n$ equations in (1a) together. This yields the interesting result that

$$
\sum_{i=1}^{n} \frac{1}{x_{i}{ }^{a}}=n .
$$

This is true for all $a$ and it can be interpreted as giving the total 'force' experienced by the particle at $x=0$ due to repulsion from other particles.

Taking (29), we now split the sum into two sums, one in which the inner scaling is appropriate and a second in which the outer scaling is appropriate. As in any problem involving matched asymptotic expansions, there will be no such abrupt transition from the inner region to the outer region; instead, we choose our transition point, $x_{K}$, to be somewhere in the intermediate (or overlap) region where $1 \ll x_{K} \ll n^{\frac{1}{a}}$. Thus,

$$
n^{\frac{a}{1-\kappa}-1} \sum_{i=1}^{K} \frac{1}{\eta_{i}^{a}}+n^{-1} \sum_{i=K+1}^{n} \frac{1}{\xi_{i}^{a}}=n .
$$

The second sum in $(30)$ can be replaced by an integral. Noting that $\xi_{K} \sim K^{\frac{1}{1-\kappa}} n^{-\frac{1}{1-\kappa}}$, we 
obtain

$$
n^{-1} \sum_{i=K+1}^{n} \frac{1}{\xi_{i}{ }^{a}}=\int_{\xi_{K+1}}^{\xi_{n}} \frac{\rho(\xi)}{\xi^{a}} d \xi+\mathcal{O}\left(K^{-\frac{a}{1-\kappa}} n^{\frac{a}{1-\kappa}-1}\right) .
$$

Assuming $a+\kappa>1$, the dominant contribution to this integral will come from a region near $\xi=\xi_{K+1}$. Using (27) to approximate $\rho$ in this area, we ultimately find that

$$
n^{-1} \sum_{i=K+1}^{n} \frac{1}{\xi_{i}^{a}}=\frac{1-\kappa}{a+\kappa-1} B^{-a} K^{1-\frac{a}{1-\kappa}} n^{\frac{a}{1-\kappa}-1}+o\left(n^{\frac{a}{1-\kappa}-1}\right) .
$$

Substituting this into (30) yields

$$
n^{\frac{a}{1-\kappa}-1} \sum_{i=1}^{K} \frac{1}{\eta_{i}^{a}}+\frac{1-\kappa}{a+\kappa-1} B^{-a} K^{1-\frac{a}{1-\kappa}} n^{\frac{a}{1-\kappa}-1}+o\left(n^{\frac{a}{1-\kappa}-1}\right)=n .
$$

As both terms on the left-hand side must be positive, we find that balance is only achieved when $\kappa=1-\frac{a}{2}$. This is consistent with the observation that $\rho \sim \xi^{-\frac{1}{2}}$ when $a=1$. If we had taken $a+\kappa<1$ above, we would have found that the integral in (31) is an order-one term. This would again lead to $\kappa=1-\frac{a}{2}$, in contradiction with the assumption $a+\kappa<1$.

Given $\kappa=1-\frac{a}{2}$, the correct rescaling for the inner region is given by $\eta=\xi n^{\frac{2}{a}}=x n^{\frac{1}{a}}$. To obtain the leading-order problem in the inner region, we take (1) and split the sum at $K$ as before. This yields

$$
\begin{gathered}
n \sum_{j=0, i \neq j}^{K} \frac{\operatorname{sgn}\left(\eta_{i}-\eta_{j}\right)}{\left|\eta_{i}-\eta_{j}\right|^{a}}-n^{-1} \sum_{j=K+1}^{n} \frac{1}{\left(\xi_{j}-\eta_{i} n^{-\frac{2}{a}}\right)^{a}}=1 . \\
\eta_{0}=0 .
\end{gathered}
$$

Note that this system will only be valid in a region where $\xi \sim n^{-\frac{1}{a}}$ and hence $i \sim n^{\frac{1}{2}}$.

We now repeat the process described by Voskoboinikov et al. [6] for $a=1$. Approximating the second sum in (33a) with an integral, we obtain

$$
n \sum_{j=0, i \neq j}^{K} \frac{\operatorname{sgn}\left(\eta_{i}-\eta_{j}\right)}{\left|\eta_{i}-\eta_{j}\right|^{a}}-\int_{\xi_{K+1}}^{\xi_{n}} \frac{\rho\left(\xi^{\prime}\right)}{\left(\xi^{\prime}-\eta_{i} n^{-\frac{2}{a}}\right)^{a}} d \xi^{\prime}=1+o(1) .
$$


Using (9), this implies

$$
n \sum_{j=0, i \neq j}^{K} \frac{\operatorname{sgn}\left(\eta_{i}-\eta_{j}\right)}{\left|\eta_{i}-\eta_{j}\right|^{a}}=f_{0}^{\xi_{K+1}} \frac{\operatorname{sgn}\left(\xi^{\prime}-\eta_{i} n^{-\frac{2}{a}}\right)}{\left(\xi^{\prime}-\eta_{i} n^{-\frac{2}{a}}\right)^{a}} \rho\left(\xi^{\prime}\right) d \xi^{\prime}+o(1) .
$$

Since $\rho=A \xi^{-\left(1-\frac{a}{2}\right)}+o\left(\xi^{-\left(1-\frac{a}{2}\right)}\right)$, we find that

$$
\begin{aligned}
f_{0}^{\xi_{K+1}} \frac{\operatorname{sgn}\left(\xi^{\prime}-\eta_{i} n^{-\frac{2}{a}}\right)}{\left(\xi^{\prime}-\eta_{i} n^{-\frac{2}{a}}\right)^{a}} \rho\left(\xi^{\prime}\right) d \xi^{\prime} & \sim A f_{0}^{\xi_{K+1}} \frac{\operatorname{sgn}\left(\xi^{\prime}-\eta_{i} n^{-\frac{2}{a}}\right)}{\left(\xi^{\prime}-\eta_{i} n^{-\frac{2}{a}}\right)^{a}}\left(\xi^{\prime}\right)^{-\left(1-\frac{a}{2}\right)} d \xi^{\prime} \\
& \sim A n f_{0}^{\xi_{K+1} n^{\frac{2}{a}}} \frac{\operatorname{sgn}\left(\eta^{\prime}-\eta_{i}\right)}{\left(\eta^{\prime}-\eta_{i}\right)^{a}}\left(\eta^{\prime}\right)^{-\left(1-\frac{a}{2}\right)} d \eta^{\prime} .
\end{aligned}
$$

This integral can be expressed exactly in terms of hypergeometric functions. Given that $\xi_{K+1} n^{\frac{2}{a}} \gg \eta_{i}$, we ultimately find that

$$
f_{0}^{\xi_{K+1} n^{\frac{2}{a}}} \frac{\operatorname{sgn}\left(\eta^{\prime}-\eta_{i}\right)}{\left(\eta^{\prime}-\eta_{i}\right)^{a}}\left(\eta^{\prime}\right)^{-\left(1-\frac{a}{2}\right)} d \eta^{\prime}=\mathcal{O}\left(\left(\xi_{K+1}\right)^{v} n^{\frac{2 v}{a}}\right),
$$

where $v=\max \left[-\left(1-\frac{a}{2}\right),-\frac{a}{2}\right]$.

Thus, (34) becomes

$$
\sum_{j=0, i \neq j}^{K} \frac{\operatorname{sgn}\left(\eta_{i}-\eta_{j}\right)}{\left|\eta_{i}-\eta_{j}\right|^{a}}=\mathcal{O}\left(K^{\frac{2 v}{a}}\right)
$$

and, in the limit $n \rightarrow \infty$ (and hence $K \rightarrow \infty$ ), we find that the leading-order system for $\left\{\eta_{i}\right\}$ is

$$
\begin{gathered}
\sum_{j=0, i \neq j}^{\infty} \frac{\operatorname{sgn}\left(\eta_{i}-\eta_{j}\right)}{\left|\eta_{i}-\eta_{j}\right|^{a}}=0 \\
\eta_{0}=0
\end{gathered}
$$

Like the system investigated by Voskoboinikov et al. [6], this system is scale invariant and it is necessary to use a discrete-continuous analogue of Van Dyke's matching condition to supply the additional information required. As the outer solution near $\xi=0$ is given by 
(28), we find that our complete inner problem takes the form

$$
\sum_{j=0, i \neq j}^{\infty} \frac{\operatorname{sgn}\left(\eta_{i}-\eta_{j}\right)}{\left|\eta_{i}-\eta_{j}\right|^{a}}=0
$$

subject to

$$
\begin{gathered}
\eta_{0}=0, \\
\eta_{i} \rightarrow B i^{\frac{2}{a}} \text { as } i \rightarrow \infty .
\end{gathered}
$$

From Voskoboinikov et al. [6], we find that $B=\frac{\pi^{2}}{8}$ when $a=1$. However, we have so far been unable to construct an expression for $B$ that is valid for general $a \in(0,2)$; this would be easily obtained if a closed-form solution to (9) were known. Instead, we define $\left\{\hat{\eta}_{i}\right\}$ to be the solution to the normalised inner problem,

$$
\sum_{j=0, i \neq j}^{\infty} \frac{\operatorname{sgn}\left(\hat{\eta}_{i}-\hat{\eta}_{j}\right)}{\left|\hat{\eta}_{i}-\hat{\eta}_{j}\right|^{a}}=0
$$

subject to

$$
\begin{gathered}
\hat{\eta}_{0}=0, \\
\hat{\eta}_{i} \rightarrow i^{\frac{2}{a}} \text { as } i \rightarrow \infty .
\end{gathered}
$$

It follows that

$$
\eta_{i}=B \hat{\eta}_{i}
$$

and thus $x_{i} n^{\frac{1}{a}} \approx B \hat{\eta}_{i}$, for small $i$ and large $n$.

Substituting (37) into (32) and taking $K \rightarrow \infty$, we find that

$$
\sum_{i=1}^{\infty} \frac{1}{\hat{\eta}_{i}^{a}}=B^{a}
$$

Importantly, this means that we can easily use the solution to (36) to obtain $B$. 
We have only been able to solve (36) numerically. To obtain numerical solutions, we truncate the system at some large value $i=P$ and assume that all particles beyond $\hat{\eta}_{P}$ are spaced so that

$$
\hat{\eta}_{P+k}-\hat{\eta}_{P}=(P+k)^{\frac{2}{a}}-P^{\frac{2}{a}} .
$$

Thus, we effectively replace equations (36a) and (36c) with

$$
\sum_{j=0, i \neq j}^{P} \frac{\operatorname{sgn}\left(\hat{\eta}_{i}-\hat{\eta}_{j}\right)}{\left|\hat{\eta}_{i}-\hat{\eta}_{j}\right|^{a}}-\sum_{k=1}^{R} \frac{1}{\left((P+k)^{\frac{2}{a}}-P^{\frac{2}{a}}+\hat{\eta}_{P}-\hat{\eta}_{i}\right)^{a}}=0
$$

where $R$ is a suitably large number. We solve this system using a Newton iteration scheme with a starting condition of $\hat{\eta}_{i}=i^{\frac{2}{a}}$.

Importantly, the system in (35) is independent of $n$. Hence, (36) only needs to be solved once for any given value of $a$ in order to obtain approximate locations of the first few particles in the boundary layer. As shown in Figure 4, these asymptotic results compare well with the particle positions obtained by rescaling the solutions to the full problem in (1).

\subsection{Boundary layer near $\xi=0$ for $a>2$}

We now consider the boundary layer near $\xi=0$ for $a>2$. In this case, the fact that the leading-order density in (17) remains order-one as $\xi \rightarrow 0$ implies that $\xi_{i+1}-\xi_{i} \sim \mathcal{O}\left(n^{-1}\right)$ throughout the inner region. Hence, we introduce our inner spatial variable, $\eta$, so that $\eta=\xi n=x n^{\frac{1}{a}}$.

Returning to the original system of equations in (1), we again split the sum at some $K$ chosen so that $n^{\frac{1}{a}} \ll K \ll n$ and hence $x_{K}$ lies in the intermediate matching region. Thus,

$$
n \sum_{j=0, i \neq j}^{K} \frac{\operatorname{sgn}\left(\eta_{i}-\eta_{j}\right)}{\left|\eta_{i}-\eta_{j}\right|^{a}}-n^{-(a-1)} \sum_{j=K+1}^{n} \frac{1}{\left(\xi_{j}-\eta_{i} n^{-1}\right)^{a}}=1,
$$




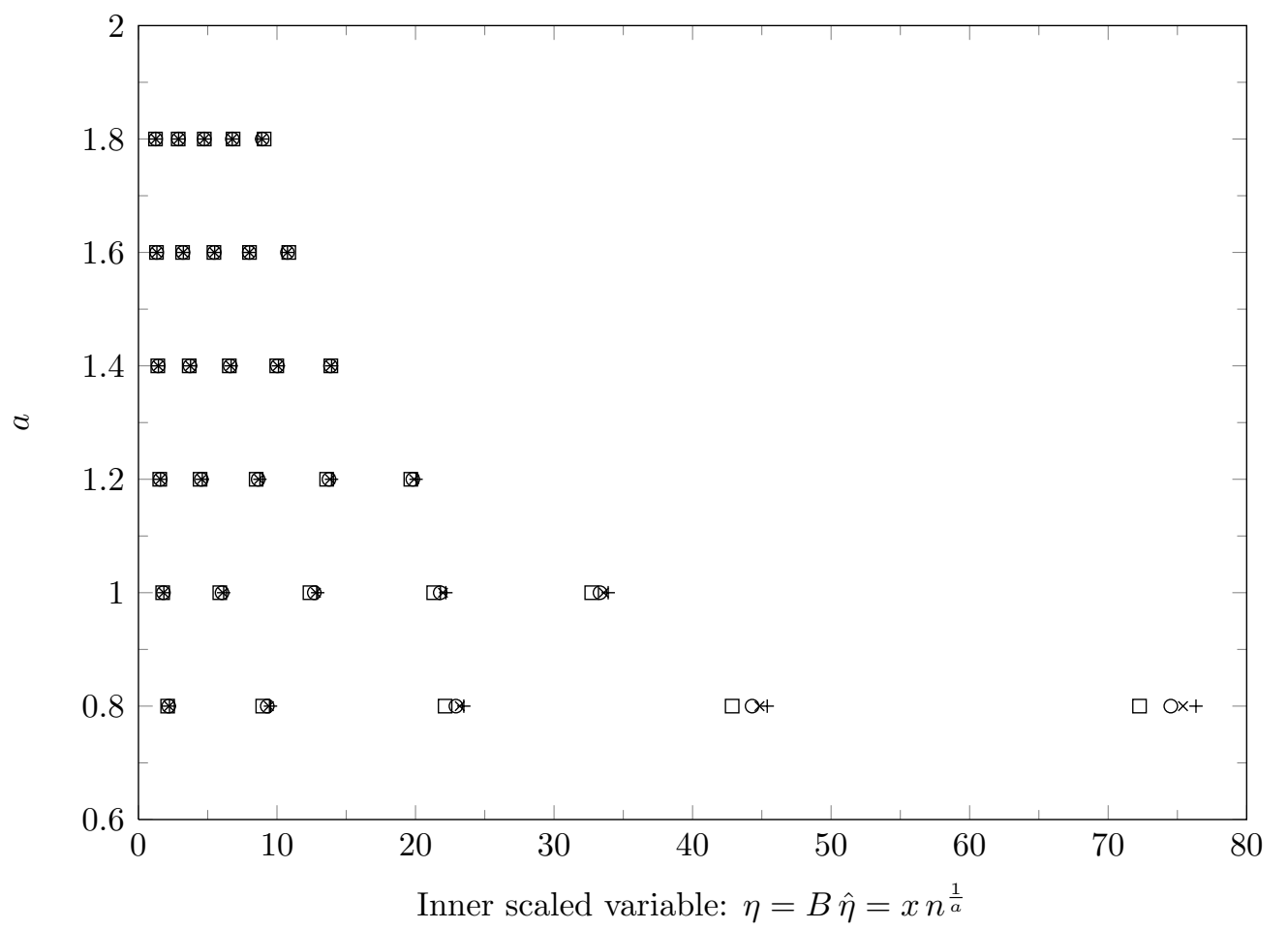

Figure 4: Rescaled locations of the first five particles obtained from numerical solution of the full problem compared with solutions to the asymptotic problem in (36) for $a=0.8, a=1, a=1.2$, $a=1.4, a=1.6$ and $a=1.8$. In each case, the asymptotic solution is shown as + , the numerical solution for $n=20$ is shown as $\square$, the numerical solution for $n=50$ is shown as $\bigcirc$, and the numerical solution for $n=100$ is shown as $\times$. Note that the value of $B$ is obtained using (38). 
for all $i \sim n^{\frac{1}{a}}$. Approximating the second sum using an integral, we obtain

$$
\begin{aligned}
n \sum_{j=0, i \neq j}^{K} \frac{\operatorname{sgn}\left(\eta_{i}-\eta_{j}\right)}{\left|\eta_{i}-\eta_{j}\right|^{a}}- & n^{-(a-2)} \int_{\xi_{K+1}}^{\xi_{n}} \frac{\rho\left(\xi^{\prime}\right)}{\left(\xi^{\prime}-\eta_{i} n^{-1}\right)^{a}} d \xi^{\prime} \\
& +n^{-(a-1)}\left(\frac{1}{2\left(\xi_{K+1}-\eta_{i} n^{-1}\right)^{a}}+\frac{1}{2\left(\xi_{n}-\eta_{i} n^{-1}\right)^{a}}+\ldots\right)=1 .
\end{aligned}
$$

Since $K \gg n^{\frac{1}{a}}$, we find that

$$
n^{-(a-1)}\left(\frac{1}{2\left(\xi_{K+1}-\eta_{i} n^{-1}\right)^{a}}\right) \sim o(1)
$$

Moreover, the dominant contribution to the integral in (41) will come from its lower bound and will also be of this size. Hence, (41) yields

$$
\sum_{j=0, i \neq j}^{K} \frac{\operatorname{sgn}\left(\eta_{i}-\eta_{j}\right)}{\left|\eta_{i}-\eta_{j}\right|^{p}}=\mathcal{O}\left(n^{-1}\right) .
$$

In the limit as $n$ (and hence $K$ ) tends to infinity, this means that the leading-order inner problem again takes the form

$$
\begin{gathered}
\sum_{j=0, j \neq i}^{\infty} \frac{\operatorname{sgn}\left(\eta_{i}-\eta_{j}\right)}{\left|\eta_{i}-\eta_{j}\right|^{a}}=0, \quad i=1,2, \ldots \\
\eta_{0}=0 .
\end{gathered}
$$

As above, (42) is scale-invariant and we use a discrete-continuous analogue of Van Dyke's matching condition to supply the additional information required. Noting from (17) that

$$
\lim _{\xi \rightarrow 0} \rho_{0}(\xi)=\zeta(a-1)^{-\frac{1}{a}}
$$


we ultimately find that the full system to be solved is

$$
\sum_{j=0, j \neq i}^{\infty} \frac{\operatorname{sgn}\left(\eta_{i}-\eta_{j}\right)}{\left|\eta_{i}-\eta_{j}\right|^{a}}=0, \quad i=1,2, \ldots
$$

subject to the conditions

$$
\begin{gathered}
\eta_{0}=0 \\
\lim _{i \rightarrow \infty}\left[\eta_{i+1}-\eta_{i}\right]=\zeta(a-1)^{\frac{1}{a}} .
\end{gathered}
$$

As before, we have only been able to solve this system numerically. To obtain numerical solutions, we truncate the system at some large value $i=P$ and assume that all particles beyond $\eta_{P}$ are evenly spaced in accordance with condition (43c). Thus, we effectively replace (43a) and (43c) with

$$
\sum_{j=0, j \neq i}^{P} \frac{\operatorname{sgn}\left(\eta_{i}-\eta_{j}\right)}{\left|\eta_{i}-\eta_{j}\right|^{a}}-\sum_{k=1}^{\infty} \frac{1}{\left(\eta_{P}+\zeta(a-1)^{\frac{1}{a}} k-\eta_{i}\right)^{a}}=0, \quad i=1,2, \ldots, P
$$

As with the full problem, Newton iteration is used to solve this system of algebraic equations; in this case, we use a starting condition in which the particles are evenly spaced at intervals of $\zeta(a-1)^{\frac{1}{a}}$.

As before, the system in (43) is independent of $n$ and hence it only needs to be solved once for any given value of $a$. In Figure 5, we see that there is again good agreement between this asymptotic solution and numerical solutions to the full problem in (1).

\subsection{Boundary layer near $\xi=\xi^{*}$ for $a>2$}

There are some interesting differences between the boundary layer near $\xi^{*}$ and the boundary layer near zero. As for the boundary layer near $\xi=0$ for $a<2$, it is necessary to integrate 


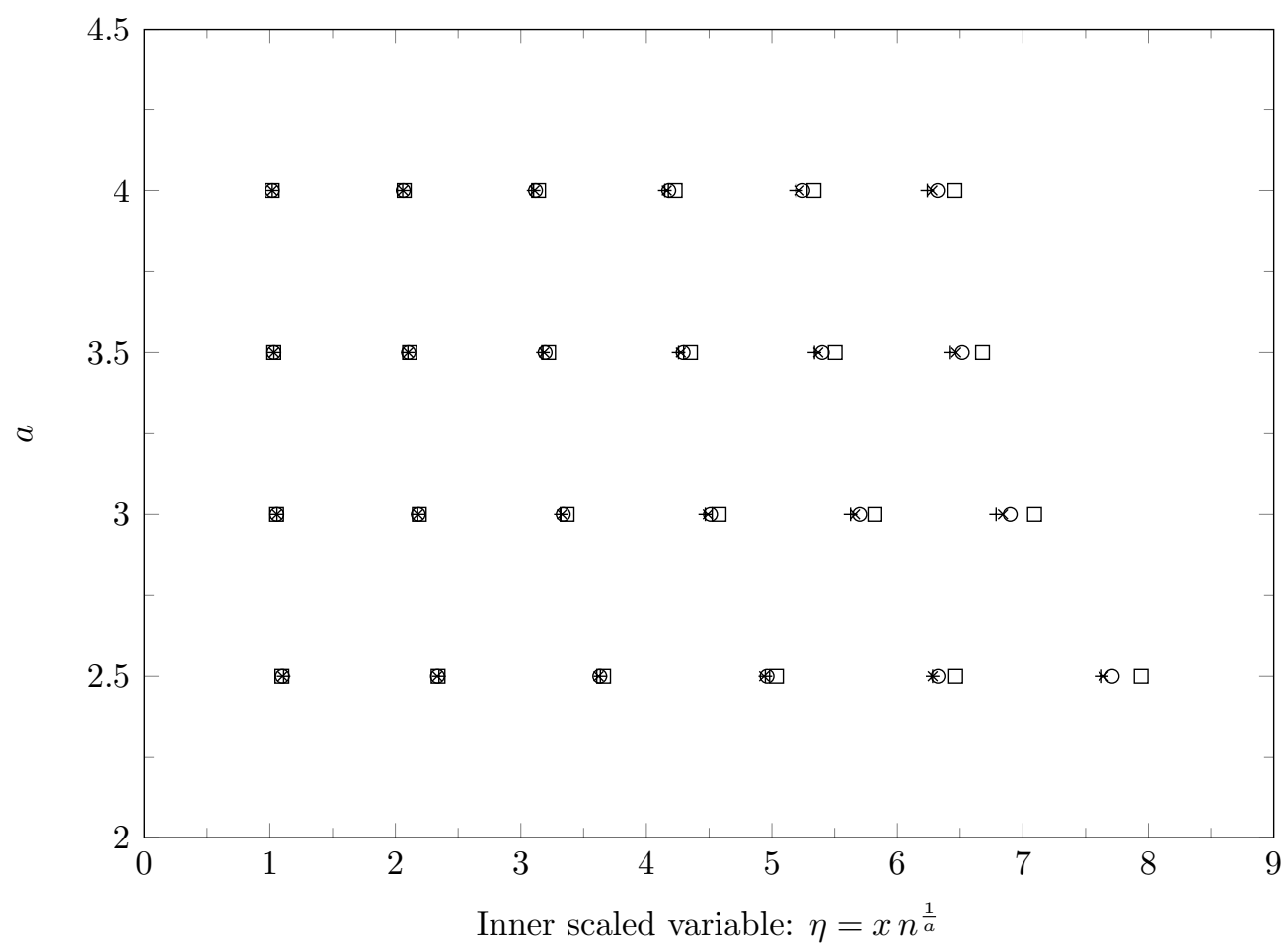

Figure 5: Rescaled locations of the first six particles obtained from numerical solution of the full problem compared with solutions to the asymptotic problem in (43) for $a=2.5, a=3, a=3.5$ and $a=4$. In each case, the asymptotic solution is shown as + , the numerical solution for $n=20$ is shown as $\square$, the numerical solution for $n=50$ is shown as $\bigcirc$, and the numerical solution for $n=100$ is shown as $\times$. 
the solution to the outer probem in order to obtain the correct scaling. In this case,

$$
\left(\frac{a-1}{a \zeta(a-1)}\right)^{\frac{1}{a-1}} \int_{\xi_{n-i}}^{\xi^{*}}\left(\xi^{*}-\xi\right)^{\frac{1}{a-1}} d \xi=\frac{i}{n}\left(1+\mathcal{O}\left(n^{u}\right)\right),
$$

where $u=\max [-1,-(a-2)]$ as before. Hence,

$$
\xi^{*}-\xi_{n-i}=\frac{a \zeta(a-1)^{\frac{1}{a}} i^{\frac{a-1}{a}}}{a-1} n^{-\frac{a-1}{a}}\left(1+\mathcal{O}\left(n^{u}\right)\right) .
$$

This leads us to introduce a rescaled spatial variable, $\chi$, so that

$$
\chi=\left(\xi^{*}-\xi\right) n^{\frac{a-1}{a}}=\xi^{*} n^{\frac{a-1}{a}}-x .
$$

We also renumber the particles so that $\chi_{i}$ corresponds to the same particle as $\xi_{n-i}$.

As before, we choose some $K$ so that $n^{\frac{1}{a}} \ll K \ll n$ and thus $x_{n-K}$ lies in the intermediate region. Applying the inner scaling for $j \leq K$ and considering $i \sim \mathcal{O}\left(n^{\frac{1}{a}}\right)$, we split the sum in (1a) to obtain

$$
-\sum_{j=0, j \neq i}^{K} \frac{\operatorname{sgn}\left(\chi_{i}-\chi_{j}\right)}{\left|\chi_{i}-\chi_{j}\right|^{a}}+n^{-(a-1)} \sum_{j=K+1}^{n} \frac{1}{\left(\xi^{*}-\chi_{i} n^{-\frac{a-1}{a}}-\xi_{n-j}\right)^{a}}=1 .
$$

Since $K \gg n^{\frac{1}{a}}$, we find that

$$
n^{-(a-1)} \sum_{j=K+1}^{n} \frac{1}{\left(\xi^{*}-\chi_{i} n^{-\frac{a-1}{a}}-\xi_{n-j}\right)^{a}}=o(1)
$$

In the limit $n \rightarrow \infty$, (45) therefore yields

$$
\sum_{j=0, j \neq i}^{\infty} \frac{\operatorname{sgn}\left(\chi_{i}-\chi_{j}\right)}{\left|\chi_{i}-\chi_{j}\right|^{a}}=-1, \quad i=0,1,2, \ldots
$$

The system in (46) is translation-invariant and we can supply the missing information using Van Dyke's matching rule. This is an interesting contrast from the scale-invariant equations encountered in the boundary layer near $\xi=0$. Using (44), we ultimately find that our full 
problem for the boundary layer near $\xi^{*}$ is

$$
\sum_{j=0, j \neq i}^{\infty} \frac{\operatorname{sgn}\left(\chi_{i}-\chi_{j}\right)}{\left|\chi_{i}-\chi_{j}\right|^{a}}=-1, \quad i=0,1,2, \ldots
$$

subject to

$$
\chi_{i} \rightarrow \frac{a}{a-1} \zeta(a-1)^{\frac{1}{a}} i^{\frac{a-1}{a}}, \text { as } i \rightarrow \infty .
$$

As with the earlier systems, we have only been able to solve (47) numerically. Again, this requires us to truncate the system at some large value $i=P$, yielding

$$
\sum_{j=0, j \neq i}^{P} \frac{\operatorname{sgn}\left(\chi_{i}-\chi_{j}\right)}{\left|\chi_{i}-\chi_{j}\right|^{a}}+\sum_{j=P+1}^{\infty} \frac{1}{\left(\frac{a}{a-1} \zeta(a-1)^{\frac{1}{a}} j^{\frac{a-1}{a}}-\chi_{i}\right)^{a}}=-1, \quad i=0,1,2, \ldots, P
$$

A Newton iteration scheme with a starting condition of $\chi_{i}=\frac{a}{a-1} \zeta(a-1)^{\frac{1}{a}} i^{\frac{a-1}{a}}$ gives the results shown in Figure 6, where comparisons are made between $\frac{\xi_{n}}{\xi^{*}}$ from the full problem and asymptotic approximations based on

$$
\xi_{n} \sim \xi^{*}-\chi_{0} n^{-\frac{a-1}{a}} .
$$

It is interesting to note that the method described in this section should also be applicable to the boundary layer near $\xi=\xi^{*}$ for $0<a<2$. If a solution to (9) were known, it could be integrated to obtain the correct scaling near $\xi=\xi^{*}$. Repeating the process above would then yield an infinite system and associated matching condition similar to (47).

\section{Discussion}

One of the most interesting features of this problem is the transition between the singular integral equation for $0<a<2$ and the differential equation for $a>2$. When $a$ is small, the repulsive effect of each particle decreases slowly with distance. As a result, each particle effectively feels every other particle and the particle density at equilibrium is dominated 


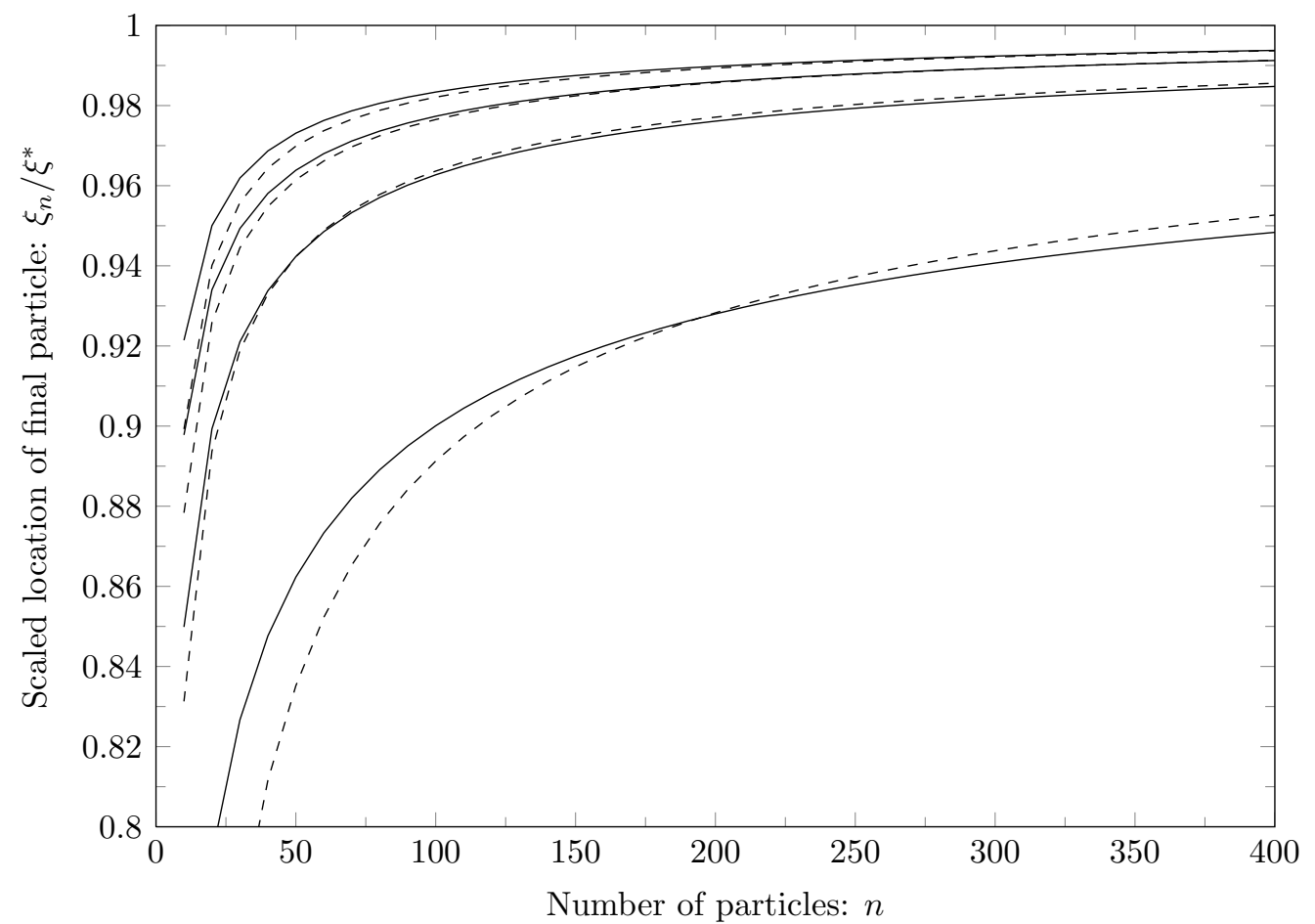

Figure 6: Numerically calculated values for $\xi_{n} / \xi^{*}$ from the full problem, shown as continuous lines, compared with asymptotic approximations of $\xi_{n} / \xi^{*}$ obtained from (47), shown as dashed lines. From bottom to top, the plot shows results for $a=2.5, a=3, a=3.5$ and $a=4$. 
by nonlocal effects. This leads to a singular integral equation for the particle density, (9). In contrast, when $a$ is large, the repulsion from each particle drops off very rapidly as a function of distance. Hence, each particle only feels its nearest neighbours and the density at equilibrium is dominated by the local environment of each particle. This gives rise to a differential equation in which the density far from any given point is no longer important.

Although these observations explain the transition between the two equations in broad terms, it is interesting to consider why the change occurs at $a=2$. At first, it might be expected that the transition should occur at $a=1$ because this corresponds to a non-integrable singularity appearing in the integral equation. However, the transition point is shifted by the fact that the ability to define a density function implies that the particles are almost evenly spaced. As a result, the highest-order local effects of a particle's nearest neighbours cancel each other out. This can be seen in the derivation of the $0<a<2$ equation through the fact that we were able to combine two opposed terms from the Euler-Maclaurin error expression in (7) to give an error term that was $\mathcal{O}\left(n^{-(2-a)}\right)$ instead of $\mathcal{O}\left(n^{-(1-a)}\right)$. Similarly, the even spacing allowed us to pair particles in the derivation of the $a>2$ and $a=2$ equations, yielding terms that were $\mathcal{O}\left(n^{a-1-a q}\right)$ instead of $\mathcal{O}\left(n^{a-a q}\right)$.

This counterintuitive result reveals the importance of approaching this problem from the discrete system in (1). An alternative would have been to start from the force function, $F(x)$, which represents the force experienced by a hypothetical particle inserted into the system at the point $x$. This is defined as

$$
F(x)=-1+\sum_{j=0}^{n} \frac{\operatorname{sgn}\left(x-x_{j}\right)}{\left|x-x_{j}\right|^{a}} .
$$

Rescaling $x$ with $x_{n}$ to get $\xi$, it would appear appropriate to approximate $F(x)$ with an integral over the particle density, $\rho(\xi)$, and then take the regular part of this integral to be zero throughout the domain of interest. Effectively, this is the method used by Voskoboinikov et al. $[5,4]$ in their investigation of a dislocation pile-up against a bimetallic interface. Although this is appropriate when $a=1$, it makes it difficult to see the transition to a differential equation as $a$ increases. Indeed, a naïve use of this method struggles to recover the correct equation when $a>1$ because it is hard to see that the transition between regimes occurs at $a=2$ rather than $a=1$. 
A further interesting feature of the transition between the integral equation and the differential equation is that (23), the equation for $a=2$, is quite different from both (9) and (15). In order to elucidate the connection between these three distinct cases, consider the following sum, representing a generalised harmonic number:

$$
H_{b}(n)=\sum_{i=1}^{n} \frac{1}{i^{b}}
$$

As described in Appendix A, this sum is characterised by different asymptotic behaviour for $n \rightarrow \infty$ depending on whether $b$ is greater or less than one and depending on whether $|b-1| \log n$ is large or small.

In much the same way, our problem can be separated into three different cases depending on whether $a$ is greater or less than two and on whether $|a-2| \log n$ is large or small. To see this last distinction, we note that the correction term to both (9) and (15) is $\mathcal{O}\left(n^{-|a-2|}\right)$; thus, these equations are inappropriate as $a \rightarrow 2$ if $|a-2| \log n$ is small.

In Appendix $\mathrm{A}$, we show that a perturbation around $b=1$ can be used to construct a mathematical bridge between the three types of asymptotic behaviour for the generalised harmonic numbers. Proposing an expansion in powers of $(b-1) \log n$, we find that these terms combine to form a term that is exponentially large when $b<1$ and exponentially small when $b>1$. Similarly, we can perturb around $a=2$ in our main problem to obtain corrections to $(24)$ in powers of $(a-2) \log n$. We conjecture that taking infinitely many of these terms may enable us to build a similar connection between the three types of behaviour observed.

\section{Conclusions}

We have presented an asymptotic analysis of an elementary problem involving the equilibrium of a large number of mutually-repelling particles. In practical applications, these 'particles' could be any one of a variety of physical entities, including dislocation monopoles, dislocation dipoles, line charges, point charges etc. In each case, the essential feature is that the repulsion between particles is inversely proportional to some power of the distance between particles. 
If the repulsion drops off very quickly with distance, local effects dominate, leading to a differential equation for the particle density. Alternatively, if the repulsion decreases slowly, non-local effects are important and we obtain an integral equation. The transition between these two regimes occurs at $a=2$ (i.e. inverse square repulsion between particles), and this case needs to be treated carefully. For all values of $a$, boundary layers are observed and it is necessary to use matched asymptotic techniques to go from the continuous problem in the outer region to discrete problems near the boundaries.

Several open problems still remain to be resolved. Most notably, we have been unable to obtain a closed-form solution to the integral equation in (9) nor have we characterised the boundary layers for $a=2$. Also, we observe that some of the methods described are only relevant to one-dimensional problems; it would be interesting to investigate the techniques required to construct continuum approximations of higher-dimensional equivalents of the system studied here.

\section{Acknowledgements}

This publication is based on work supported by Award No. KUK-C1-013-04, made by King Abdullah University of Science and Technology (KAUST).

\section{References}

[1] K. C. A. Blasdale. Arrays of dislocation dipoles in cadmium. Physica Status Solidi, 21(2):649-660, 1967.

[2] G. F. Carrier, M. Krook, and C. E. Pearson. Functions of a Complex Variable - Theory and Technique. SIAM, 2005.

[3] J. P. Hirth and J. Lothe. Theory of Dislocations. John Wiley \& Sons, 2nd edition, 1982. 
[4] R. Voskoboinikov, S. Chapman, J. Mcleod, and J. Ockendon. Asymptotics of edge dislocation pile-up against a bimetallic interface. Mathematics and Mechanics of Solids, 14(1-2):284 - 295, 2009.

[5] R. E. Voskoboinikov, S. J. Chapman, and J. R. Ockendon. Continuum and discrete models of dislocation pile-ups. II. Pile-up of screw dislocations at the interface in a bimetallic solid. Philosophical Magazine Letters, 87(9):669-676, 2007.

[6] R. E. Voskoboinikov, S. J. Chapman, J. R. Ockendon, and D. J. Allwright. Continuum and discrete models of dislocation pile-ups. I. Pile-up at a lock. Journal of the Mechanics and Physics of Solids, 55(9):2007 - 2025, 2007.

\section{A Asymptotic behaviour of generalised harmonic num- bers}

The generalised harmonic numbers, $H_{b}(n)$ are defined by

$$
H_{b}(n)=\sum_{i=1}^{n} \frac{1}{i^{b}} .
$$

for all $n \in \mathbb{N}$ and $b \in \mathbb{R}$.

We are interested in the asymptotic behaviour of $H_{b}(n)$ for large $n$. Clearly, this undergoes an important change at $b=1$, as the series goes from being divergent to being convergent as $n \rightarrow \infty$. Hence, we will focus on the case where $n \rightarrow \infty$ and $b \rightarrow 1$.

In this case, we find that there are three different domains of asymptotic behaviour. When $b<1$ and $|b-1| \log n$ is large, $H_{b}(n)$ can be approximated by using an Euler-Maclaurin series. This yields

$$
H_{b}(n) \sim \frac{n^{1-b}}{1-b}-\frac{1}{1-b}+\mathcal{O}(1) .
$$

Alternatively, when $b>1$ and $|b-1| \log n$ is large, the sum can be approximated by starting from the convergent infinite sum and subtracting off the terms from $i=n+1$ onwards. This 
yields

$$
H_{b}(n) \sim \zeta(b)+\frac{n^{-(b-1)}}{b-1}+\mathcal{O}\left(n^{-b}\right) .
$$

Lastly, if $|b-1| \log n$ is small, it is appropriate to expand the sum as follows:

$$
\begin{aligned}
H_{b}(n) & =\sum_{i=1}^{n} \frac{1}{i^{b}} \\
& =\sum_{i=1}^{n} \frac{1}{i} e^{-(b-1) \log i} .
\end{aligned}
$$

Expanding this exponential function as a Taylor series, we find that

$$
\begin{aligned}
H_{b}(n) & \sim \sum_{i=1}^{n} \frac{1}{i}(1-(b-1) \log i+\ldots) \\
& \sim \log n+\gamma-\frac{(b-1)(\log n)^{2}}{2}-(b-1) \gamma_{1}+\mathcal{O}\left((b-1)^{2}(\log n)^{3}, \frac{1}{n}\right) .
\end{aligned}
$$

Just as the asymptotic behaviour of the full problem depends on whether $|a-2| \log n$ is $\operatorname{large}$ or small, we find that the behaviour of this toy problem depends on whether $|b-1| \log n$ is large or small. Interestingly, despite the fact that the connection between the three regimes is not immediately obvious, the toy problem has the advantage that we can construct an asymptotic series that is uniformly valid for all $b$.

To do this, we expand the exponential in (A.2) as an infinite series and change the order of summation. This yields

$$
H_{b}(n)=\sum_{j=0}^{\infty} \sum_{i=1}^{n} \frac{(-(b-1) \log i)^{j}}{j ! i} .
$$

Using Euler-Maclaurin series to find asymptotic expressions for

$$
\sum_{i=1}^{n} \frac{(\log i)^{j}}{i}
$$


we ultimately obtain the result that

$$
H_{b}(n) \sim \zeta(b)-\frac{n^{-(b-1)}}{b-1}-\sum_{k=1}^{\infty} \frac{B_{k}}{k !}(b)_{k-1} n^{-(b-1+k)}
$$

where $B_{k}$ is the $k$ th Bernoulli number and $(b)_{k-1}=b \cdot(b+1) \cdots(b+k-2)$ is the Pochhammer symbol. Note that this reduces to Bernoulli's formula when $b$ is a negative integer.

It is interesting to observe that the $n^{-(b+1+k)}$ terms in (A.4) arose from recombining series of logarithmic terms. When $b>1$, these give rise to terms that are exponentially small in $|b-1| \log n$, while when $b<1$ they yield terms that are exponentially large in $|b-1| \log n$.

In the full problem, we expect something similar to happen. A perturbation around $a=2$ will yield various terms in powers of $|a-2| \log n$. As $|a-2| \log n$ grows larger, some of these will combine to create exponentially large terms, whle others will combine to create exponentially small terms. In this manner, we should see that the singular integral contribution is largest when $a<2$ while the differential operator contribution is largest when $a>2$, thus giving rise to the three domains observed. Further work is needed in order to confirm this conjecture. 



\section{RECENT REPORTS}

47/09 Stochastic Partial Differential Equations as priors in ensemble methods for solving inverse problems

Potsepaev

Farmer

Aziz

48/09 DifFUZZY: A fuzzy spectral clustering algorithm for complex data

Cominetti et al. sets

01/10 Fluctuations and instability in sedimentation

Guazzelli

Hinch

02/10 Determining the equation of state of highly plasticised metals from Hinch boundary velocimetry

03/10 Stability of bumps in piecewise smooth neural elds with nonlinear adaptation

Kilpatrick

Bressloff

04/10 Random intermittent search and the tug-of-war model of motordriven transport

Newby

Bressloff

05/10 Ergodic directional switching in mobile insect groups

Escudero et al.

06/10 Derivation of a dual porosity model for the uptake of nutrients by root hairs

Zygalakis

Roose

07/10 Frost heave in compressible soils

Majumdar

Peppin

Style

Sander

08/10 A volume-preserving sharpening approach for the propagation of

Reis sharp phase boundaries in multiphase lattice Boltzmann simulations

Dellar

09/10 Anticavitation and differential growth in elastic shells

Moulton

Goriely

10/10 On the mechanical stability of growing arteries

Goriely

Vandiver

11/10 Nonlinear Correction to the Euler Buckling Formula for Compressible Cylinders

De Pascalis

Destrade

Goriely

12/10 Nonlinear Morphoelastic Plates I: Genesis of Residual Stress

McMahon

Goriely

Tabor

13/10 Nonlinear Morphoelastic Plates II: Exodus to Buckled States

McMahon

Goriely

Tabor 
14/10 Analysis of Brownian dynamics simulations of reversible biomolecular reactions

Lipkova

Zygalakis

Chapman

Erban

15/10 Travelling waves in hyperbolic chemotaxis equations

Xue

Hwang

Painter

Erban

16/10 The Physics and Mechanics of Biological Systems

Goriely

Moulton

17/10 Crust formation in drying colloidal suspensions

Style

Peppin

18/10 A Mathematical Model of Tumor-Immune Interactions

Robertson-Tessi

El-Kareh

Goriely

19/10 Elastic cavitation, tube hollowing, and differential growth in plants and biological tissues

Goriely

Moulton

Vandiver

20/10 Asymptotic expressions for the nearest and furthest dislocations

Hall in a pile-up against a grain boundary

21/10 Cardiac electromechanics: the effect of contraction model on the mathematical problem and accuracy of the numerical scheme

Pathmanathan

Chapman

Gavaghan

Whiteley

22/10 Fat vs. thin threading approach on GPUs: application to stochas-

Klingbeil tic simulation of chemical reactions

Erban

Giles

Maini

Copies of these, and any other OCCAM reports can be obtained from:

Oxford Centre for Collaborative Applied Mathematics

Mathematical Institute

24 - 29 St Giles'

Oxford

OX1 3LB

England

www.maths.ox.ac.uk/occam 